\title{
Relationship Hymenoptera Parasitica (parasitoids) and Diptera (hosts). Database.
}

Carlos Henrique Marchiori ${ }^{1}$

1 Instituto Federal Goiano

Bancos de dados ou bases de dados são conjuntos de arquivos relacionados entre si com registros sobre pessoas, lugares ou coisas. São coleções organizadas de dados que se relacionam de forma a criar algum sentido e dar mais eficiência durante uma pesquisa ou estudo.

\begin{tabular}{|c|c|c|c|c|}
\hline Species of p ar asitoids & $\begin{array}{c}\text { Number of specimens } \\
\text { (Site l) }\end{array}$ & Percentage & $\begin{array}{c}\text { Number of } \\
\text { specimens } \\
\text { (Site 2) }\end{array}$ & Percentage \\
\hline \multicolumn{5}{|l|}{ Eup elmidae: } \\
\hline Anastatus sp. & 00 & 00.0 & 05 & 9.8 \\
\hline Brasema sp. & 02 & 15.4 & 00 & 0.0 \\
\hline \multicolumn{5}{|l|}{ Scelionidae: } \\
\hline Gryon gallardoi & 11 & 84.6 & 40 & 78.4 \\
\hline Trissolcus sp. & 00 & 00.0 & 06 & 11.8 \\
\hline Total & 13 & 100.0 & 51 & 100.0 \\
\hline
\end{tabular}

Figure 
Table - Number of eggs and parasitoids obtain ed by ears from 113 naturally-infesting eggs of Leptoglossus zonatus collected on maize ears from December 2001 to February 2002, in two different locations [Agron omy School Farm (Site 1) an d at Santa Maria Farm (Site 2)] in Itu mbiara County, State of Goias, Central Brazil $\left(18^{\circ} 25^{\prime} \mathrm{S} ; 49^{\circ} 13^{\prime} \mathrm{W}\right)$.

\begin{tabular}{|c|c|c|c|}
\hline Site/ total of eggs & Number of ears & Eggs & Par asitoids \\
\hline \multirow[t]{3}{*}{ Site $1-41$ eggs } & ear one & 20 & 2 Brasema sp. \\
\hline & & & 3 Gryon gallardoi \\
\hline & Ear two & 21 & 8 Gryon gallardoi \\
\hline \multirow[t]{5}{*}{ Site $2-72$ eggs } & ear one & 20 & 05 Anastatus sp. \\
\hline & & & 06 Trissolcus sp. \\
\hline & & & 01 Gryon gallardoi \\
\hline & ear two & 23 & 18 Gryon gallardoi \\
\hline & ear three & 29 & 21 Gryon gallardoi \\
\hline
\end{tabular}

Figure

Table - Hosts of Paraganaspis egeria collected in several substracts from January, 1999 to october. 2001 in Itumbiara, Goiás, Brazil.

\begin{tabular}{|c|c|c|c|c|}
\hline Substracts & Frequency & Species & Frequency & Percentage \\
\hline Pig car Carcass & 3110 & Chrysomya albiceps & 05 & 0.16 \\
\hline \multirow[t]{2}{*}{ Cattle Dung } & 1558 & Palaeosepsis spp. & 16 & 1.02 \\
\hline & 1213 & Sarcophagula occidua & 175 & 14.4 \\
\hline Human Feces & 125 & Fannia pusio & 03 & 240 \\
\hline Cattle Kidneys & 61 & Fannia pusio & 05 & 8.19 \\
\hline ChickeChicken & 20 & Fannia pusio & 02 & 10.0 \\
\hline TotalT Total & 6087 & - & 206 & - \\
\hline
\end{tabular}

Figure 
Table - Parastoids of Diptera collected in traps of different colors in the south of Goias State from March to December 2006.

\begin{tabular}{lcc}
\hline Traps color & Species of parasitoids & Frequency \\
\hline Yellow & Brachymeria podagrica & 14 \\
& Brachymeria sp. & 03 \\
Blue & Brachymeria podagrica & 04 \\
& Brachymeria sp. & 11 \\
White & Brachymeria podagrica & 10 \\
& Spalangia camerani & 02 \\
Black & Brachymeria podagrica & 37 \\
Green & & 01 \\
Total & Brachymeria podagrica & 02 \\
\hline
\end{tabular}

Figure

Table - Species of syn anthropic flies collected in humans feces, in the period from March 2001 to April 2002 in Itumbiara $\left(18^{\circ} 25^{\prime} S^{\prime}, 49^{\circ} 13^{\prime} \mathrm{W}\right)$ County, Goiás State, Central Brazil.

\begin{tabular}{|c|c|c|c|c|}
\hline$\overline{\text { Fly species }}$ & $\begin{array}{c}\text { Number of } \\
\text { pupae collected }\end{array}$ & Parasitoid species & $\begin{array}{l}\text { parasitized } \\
\text { pupae }\end{array}$ & Percentage \\
\hline \multirow[t]{2}{*}{ Fannia pusio } & 157 & Pachycrepoideus vindemmiae & 7 & 4.5 \\
\hline & & Paraganaspis egeria & 1 & 0.6 \\
\hline Musca domestica & 20 & Hemencyrtus herbertii & 5 & 25.0 \\
\hline Ophyra sp. & 06 & Brachymeria podagrica & 1 & 16.7 \\
\hline \multirow[t]{4}{*}{ Oxysarcodexia thornax } & 109 & Brachymeria podagrica & 1 & 0.9 \\
\hline & & Gnathopleura quadridentata & 22 & 20.2 \\
\hline & & Hemencyrtus herbertii & 18 & 16.5 \\
\hline & & Pachycrepoideus vindemmiae & 11 & 10.1 \\
\hline \multirow[t]{2}{*}{ Peckia chrysostoma } & 106 & Brachymeria podagrica & 19 & 17.9 \\
\hline & & Nasonia vitripennis & 5 & 4.7 \\
\hline Poecilosomella sp. & 230 & Pachycrepoideus vindemiae & 40 & 17.4 \\
\hline \multirow[t]{2}{*}{ Sarcodexia sp. } & 34 & Nasonia vitripennis & 8 & 23.5 \\
\hline & & Pachycrepoideus vindemmiae & 6 & 17.6 \\
\hline Total & 823 & & 180 & 21.9 \\
\hline
\end{tabular}

Figure 
Table - Parasitoids obtained from insects pests in southern of Goiás, Brazil

\begin{tabular}{lll}
\hline Insects pests & Specie of parasitoids & Number of specimens \\
\hline Lomonia sp. & Eupelmidae: & 04 \\
& Anastatus sp. & 03
\end{tabular}

Leptoglossus zonatus

\begin{tabular}{|c|c|c|}
\hline & Eupelmidae: & \\
\hline & Anastatus sp. & 05 \\
\hline & Brasema sp. & 02 \\
\hline & Scelionidae: & \\
\hline & Gryon gallardoi & 41 \\
\hline & Trissolcus sp. & 06 \\
\hline & Braconidae: & \\
\hline Tuta absoluta & Braconsp. & 21 \\
\hline & Earinus sp & 01 \\
\hline & Conura sp. & 13 \\
\hline Zaprionus indianus & Figitidae: & \\
\hline & Leptopilina boulardi & 03 \\
\hline & Pteromalidae: & \\
\hline & Pachycrepoideus vindemmiae & 285 \\
\hline
\end{tabular}

Figure

Table - Frequency and percentage of different parasitoid species extracted from 100 artificial cattle dung pats exposed in a pasture for different periods of time in Itumbiara, State of Goiás. Central Brazil $\left(18^{\circ} 25^{\prime} S\right.$ and $\left.49^{\circ} 13^{\prime} \mathrm{W}\right)$, from Janu ary to August 2001.

\begin{tabular}{|c|c|c|}
\hline Taxonomic group & Frequency & Percentage \\
\hline \multicolumn{3}{|l|}{ Figitidae: } \\
\hline Paraganaspis egeria & 191 & 44.4 \\
\hline \multicolumn{3}{|l|}{ Pter omalidae: } \\
\hline Spalangia drosophilae & 98 & 22.7 \\
\hline \multicolumn{3}{|l|}{ Figitidae: } \\
\hline Triplasta atrocoxalis & 51 & 11.9 \\
\hline \multicolumn{3}{|l|}{ Pter omalidae: } \\
\hline Spalangia sp. & 26 & 6.0 \\
\hline \multicolumn{3}{|l|}{ Diapriidae: } \\
\hline Trichopria sp. & 20 & 4.8 \\
\hline \multicolumn{3}{|l|}{ Pter omalidae: } \\
\hline Spalangia nigraaenea & 14 & 3.3 \\
\hline \multicolumn{3}{|l|}{ Staphylinidae: } \\
\hline Aleochara notula & 12 & 2.8 \\
\hline \multicolumn{3}{|l|}{ Pteromalidae: } \\
\hline Spalangia nigra & 06 & 1.4 \\
\hline \multicolumn{3}{|l|}{ Figitidae: } \\
\hline Triplasta coxalis & 05 & 1.2 \\
\hline \multicolumn{3}{|l|}{ Pteromalidae: } \\
\hline Spalangia endius & 03 & 0.7 \\
\hline \multicolumn{3}{|l|}{ Figitidae: } \\
\hline Neralsia splendens & 02 & 0.4 \\
\hline \multicolumn{3}{|l|}{ Figitid ae: } \\
\hline Kleidotoma nigra & 01 & 0.2 \\
\hline \multicolumn{3}{|l|}{ Pteromalidae: } \\
\hline Spalangia cameroni & 01 & 0.2 \\
\hline Total & 430 & 100 \\
\hline
\end{tabular}

Figure 
Table - Registration Gnathopieura semirufa (Brullé, 1846) parasitizing the dipterous family Sarcoph agidae listed in Itumbiara, southern Goias, using ptifall trap, in the period January at November 2005

\begin{tabular}{lllll}
\hline Diptera & $\begin{array}{l}\text { Frequency } \\
\text { Dipter ous }\end{array}$ & $\begin{array}{l}\text { Frequency } \\
\text { Parasitoid }\end{array}$ & Pupaep ar asitizing & $\begin{array}{l}\text { Percentage of } \\
\text { parasitism }\end{array}$ \\
\hline Sarcophagidae: & & 75 & 75 & 24.6 \\
Oxysarcodexia thornax & 305 & 51 & 51 & 35.7 \\
Peckia chrysostoma & 143 & & & 17.0 \\
Sarcodexia lambens & 182 & 31 & & \\
& & & & \\
Total & 630 & 157 & & \\
\hline
\end{tabular}

Figure

Table - Hosts of the parasitoid Hemencyrtus herbertii collected from March 2001 to June 2006 in the states of Goiás and Minas Gerais, Brazil.

\begin{tabular}{|c|c|c|c|}
\hline Taxonomic Group & Number of pupae & Number of pupa e para sitized & Percentage \\
\hline \multicolumn{4}{|l|}{ Calliphoridae: } \\
\hline Chrysomya albiceps & 74 & 3 & 4.1 \\
\hline Cheysomya megacephala & 76 & 1 & 1.3 \\
\hline Hemilucilia flarifacies & 16 & 3 & 18.8 \\
\hline Lucilia eximia & 268 & 3 & 1.1 \\
\hline \multicolumn{4}{|l|}{ Muscidae: } \\
\hline Euboettcheria collusor & 412 & 1 & 3.2 \\
\hline Musca domestica & 20 & 1 & 5.0 \\
\hline Ophyrasp. & 64 & 3 & 47 \\
\hline \multicolumn{4}{|l|}{ Sarcophagidae: } \\
\hline Oxysarcadexia thornax & 109 & 2 & 1.8 \\
\hline Peckia chrysostoma & 280 & 9 & 3.2 \\
\hline Sarcodexia lambens & 17 & 5 & 29.4 \\
\hline Total & 1336 & 31 & - \\
\hline
\end{tabular}

Figure 
Table - Percentage of parasitism of Musca domestica L. 1758 (Diptera: Muscidae) collected in two areas of the central region of Goiás, Brazil.

\begin{tabular}{|c|c|c|c|c|}
\hline \multirow{2}{*}{ Taxonomic Group } & \multicolumn{2}{|c|}{ Rural } & \multicolumn{2}{|c|}{ Urbana } \\
\hline & $\begin{array}{l}\text { Number of } \\
\text { pupae } \\
\text { parasitized }\end{array}$ & $\%$ parasitism & $\begin{array}{c}\text { Number of pupae } \\
\text { parasitized }\end{array}$ & $\%$ parasitism \\
\hline \multicolumn{5}{|l|}{ Encyrtidae: } \\
\hline Hemencyrtus herbertii & 5 & 1.5 & 1 & 0.2 \\
\hline \multicolumn{5}{|l|}{ Eulophidae: } \\
\hline Tachinobiasp. & - & - & 1 & 0.2 \\
\hline \multicolumn{5}{|l|}{ Pteromalidae: } \\
\hline Nasonia vitripennis & - & - & 7 & 1.5 \\
\hline Pachycrepoideus vindemmiae & 19 & 5.4 & 26 & 5.5 \\
\hline Spalangia sp. & - & - & 7 & 1.5 \\
\hline Total of pupae parasitized: & 24 & - & 42 & \\
\hline Total of pupae: & 350 & & 472 & \\
\hline
\end{tabular}

Figure 